\title{
Atmospheric neutrinos with the first KM3NeT/ORCA data and prospects for measuring the atmospheric neutrino flux
}

\author{
Dimitris Stavropoulos, ${ }^{a, b, *}$ Valentin Pestel, ${ }^{c}$ Zineb Aly, ${ }^{d}$ Ekaterini Tzamariudaki ${ }^{a}$ and \\ Christos Markou ${ }^{a}$ on behalf of the KM3NeT Collaboration \\ (a complete list of authors can be found at the end of the proceedings) \\ ${ }^{a}$ NCSR Demokritos, Institute of Nuclear and Particle Physics, Ag. Paraskevi Attikis, Athens, 15310 Greece \\ ${ }^{b}$ National Technical University of Athens, School of Applied Mathematical and Physical Sciences, \\ Zografou Campus, 9, Iroon Polytechniou str, 15780 Zografou, Athens, Greece \\ ${ }^{c}$ Nikhef, National Institute for Subatomic Physics, PO Box 41882, Amsterdam, 1009 DB Netherlands \\ ${ }^{d}$ Aix Marseille Univ, CNRS/IN2P3, CPPM, Marseille, France \\ E-mail: dstavropoulos@inp.demokritos.gr
}

\begin{abstract}
$\mathrm{KM} 3 \mathrm{NeT}$ is a research infrastructure aiming to study astrophysical sources as well as to perform particle physics studies, through the detection of neutrinos in the abyssal depths of the Mediterranean Sea. The KM3NeT/ORCA detector (Oscillation Research with Cosmics in the Abyss), currently under construction, is deployed at $2450 \mathrm{~m}$ depth near Toulon, France. It consists of vertical structures (Detection Units) equipped with spherical Digital Optical Modules, each hosting a set of photomultiplier tubes capable of detecting neutrino events from the Cherenkov radiation induced by the daughter particles. In this contribution, an analysis of data collected with the first 6 Detection Units (ORCA6) leading to a sample of atmospheric neutrino events is described. The angular resolution and the energy reconstruction performance for this event selection, which is a key factor for measuring the atmospheric neutrino flux are presented.
\end{abstract}

$37^{\text {th }}$ International Cosmic Ray Conference (ICRC 2021)

July 12th - 23rd, 2021

Online - Berlin, Germany

\footnotetext{
${ }^{*}$ Presenter
} 


\section{Introduction}

The KM3NeT Collaboration is currently constructing a research infrastructure in the depths of the Mediterranean Sea [1]. This research infrastructure consists of two detectors with different scientific goals. The ARCA (Astroparticle Research with Cosmics in the Abyss) detector is located offshore of Sicily, Italy, at a maximum depth of $3450 \mathrm{~m}$. The primary goal of this detector is to perform neutrino astronomy by detecting high energy astrophysical neutrinos $(E>\mathrm{TeV})$. The other detector, ORCA (Oscillation Research with Cosmics in the Abyss) is located $40 \mathrm{~km}$ offshore Toulon, France, at a depth of $2450 \mathrm{~m}$. The primary goal of ORCA is to measure the neutrino mass ordering, by being sensitive to oscillations of atmospheric neutrinos in the energy range of a few $\mathrm{GeV}$.

The KM3NeT/ORCA detector is an array of photomultiplier tubes (PMTs), capable of detecting neutrino events via the Cherenkov radiation emmited by the daughter particles. These 3-inch diameter PMTs are found in groups of 31, into high-resistance glass spheres called Digital Optical Modules (DOMs), in order to endure the pressure at the abyssal depths (Fig.1). The DOMs contain also the required electronics for the operation of the PMTs, the data transmission as well as instruments for the orientation, position and time calibration [2]. The DOMs are tied together in groups of 18 forming vertical structures, the so-called Detection Units (DUs - Fig.2). Each DU is anchored to the seabed and remain vertical due to the buoyancy of the DOMs as well as of a buoy which is tied on its top. The vertical distance between the DOMs in a DU is $\sim 9 \mathrm{~m}$, and the horizontal distance between the DUs is $\sim 20 \mathrm{~m}$. When completed, the ORCA detector will consist of 115 DUs, forming a cylindrical instrumented volume with a radius $\sim 100 \mathrm{~m}$, and height $\sim 160$ $\mathrm{m}$. The ARCA detector will be similar to ORCA, with a sparser instrumentation as it aims to be sensitive to higher energy neutrinos of astrophysical origin.

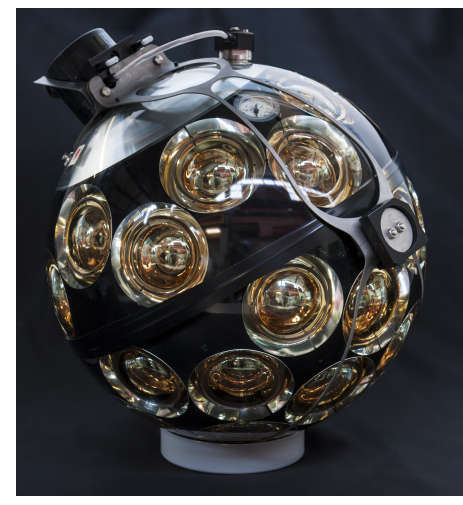

Figure 1: The Digital Optical Module, with 31 PMTs distributed on its surface.

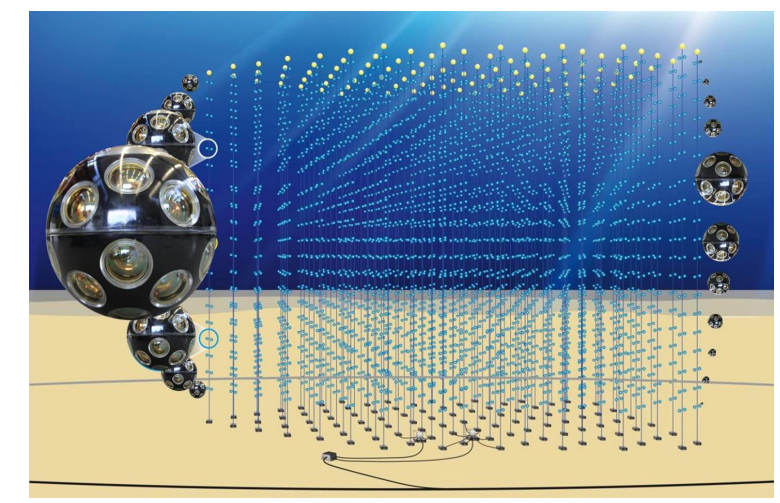

Figure 2: Artistic view of the full KM3NeT/ORCA detector. The vertical structures are the DUs on which the DOMs are attached.

The KM3NeT/ORCA detector operates with 6 DUs (ORCA6) since January 2020. In these proceedings, an analysis of data collected with ORCA6 configuration that provides a sample of atmoshperic neutrinos is presented. The angular resolution and the energy reconstruction performance of this neutrino sample as well as the prospects for a measurement of the atmospheric neutrino flux are also discussed. 


\section{Detector status and data sample}

In January 2020 two additional DUs were deployed, extending the already operational ORCA detector to the current 6-DU configuration. At present, the instrumented volume of ORCA6 is 136 kton. The layout of the ORCA6 detector is shown in Fig. 3, which depicts the footprints of the DUs on the horizontal plane.

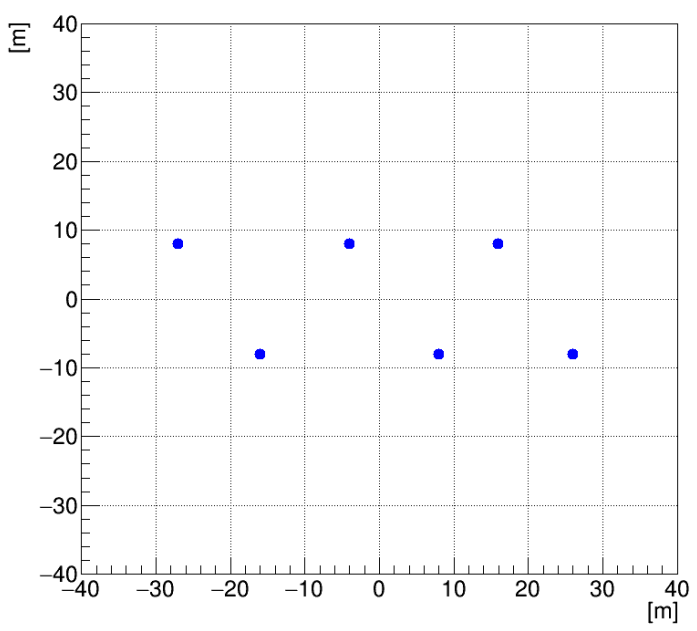

Figure 3: The DU footprints of the ORCA6 configuration.

The data used in this analysis were collected from February 11, 2020 to March 03, 2021. During this period the detector was operating $96 \%$ of the time. After a quality selection and rejection of data runs recorded for calibration and test purposes, the data sample obtained for this analysis is equivalent to 354.6 days. This corresponds to an overall $92 \%$ time efficiency with respect to the full-time period, as depicted in Fig. 4.

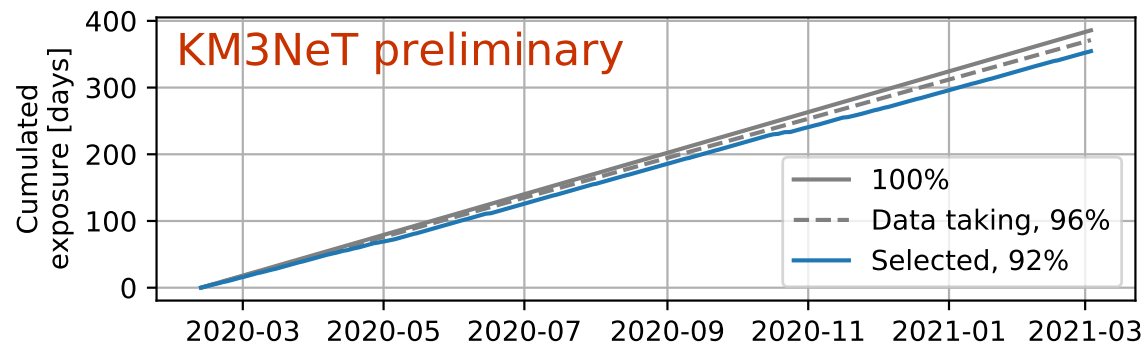

Figure 4: Cumulated exposure of overall time, data-taking time, and livetime of the selected data.

The selected data runs were processed and the events were reconstructed under the track hypothesis, using the KM3NeT software package Jpp [3]. 


\section{Simulation of atmospheric neutrinos and muons}

Events of atmospheric neutrinos and muons were simulated to evaluate the detector response. The followed strategy was to produce run-by-run simulation in which each run is simulated separately, in order to account for the variation of the detector and data-taking conditions.

For the generation of the simulated neutrino events, the gSeaGen GENIE-based software [4] was used. Neutrino and antineutrino events for charged current (CC) interactions for each flavor, as well as neutral current (NC) interaction events, were simulated. All the neutrino events were weighted using the HKKM2014 neutrino flux [5] computed for the Frejus location. Moreover, the oscillation probabilities were taken into account in the calculation of the neutrino weights. For this, the oscillation parameters were set according to NuFIT 5.0 [6], assuming normal ordering for the neutrino mass hierarchy. The atmospheric muon background events were generated using MUPAGE package [7]. The PMT response and the readout are simulated by means of a custom KM3NeT software. The digitised PMT output pulses are called as hits.

Similar to the events in data, the simulated events were reconstructed under the track hypothesis [3].

\section{Neutrino event selection}

Atmosperic neutrino events have been discriminated from the atmospheric muon background after applying several selection criteria. At first, a soft cut on the maximum value of the likelihood fit in the reconstruction procedure is applied. This is intended to reject poorly reconstructed events as well as events that are produced by noise hits.

After this pre-selection, only events with upgoing reconstructed direction are accepted. With the rejection of the reconstructed as downgoing events, the atmospheric muon background consists only of events that are mis-reconstructed. This results in a high suppression of the atmospheric muon contribution with the remaining background consisting only of events that are mis-reconstructed as upgoing. A set of selection criteria is applied then to the remaining events. These can be separated into three different categories:

- signal-like hits related criteria: The agreement between the event hits and the reconstructed track hypothesis is examined with these criteria. In more detail, a cut on the number of triggered hits which have been recorded too early with respect to what is expected according to the track hypothesis is applied; triggered hits are those that have caused the event triggering. A minimum number of DOMs with at least one hit ("signal-like hit") expected from the reconstructed track in terms of time resolution and of the distance between the emission point along the reconstructed track and the PMT position, is required.

- quality of the reconstructed event: The quality of the track reconstruction is evaluated from the likelihood function at its maximum fitted value. A cut is applied on the ratio of this likelihood value to the number of hits used in the reconstruction. Moreover, a minimum difference is also required between the likelihood of the upgoing reconstructed track and the likelihood of the best potential downgoing solution, if there is one. An additional cut on the likelihood is applied, with the minimum required likelihood value to be proportional to the 
distance between the event's reconstructed vertex position and the detector's barycenter on the horizontal plane.

- containment: The position of the reconstructed vertex of the event should be closer than 60 $\mathrm{m}$ to the barycenter of the detector, on the horizontal plane. The mean height of the triggered hits should be above the height of the event's reconstructed vertex position, as well as above $55 \mathrm{~m}$ above the seabed.

The distribution of the reconstructed energy is shown in Fig. 5 after applying the described selection criteria. A final cut is applied at this point, rejecting the events with a reconstructed energy above $100 \mathrm{GeV}$. This is to account for the limited instrumented volume of the ORCA detector at this early stage of the construction.

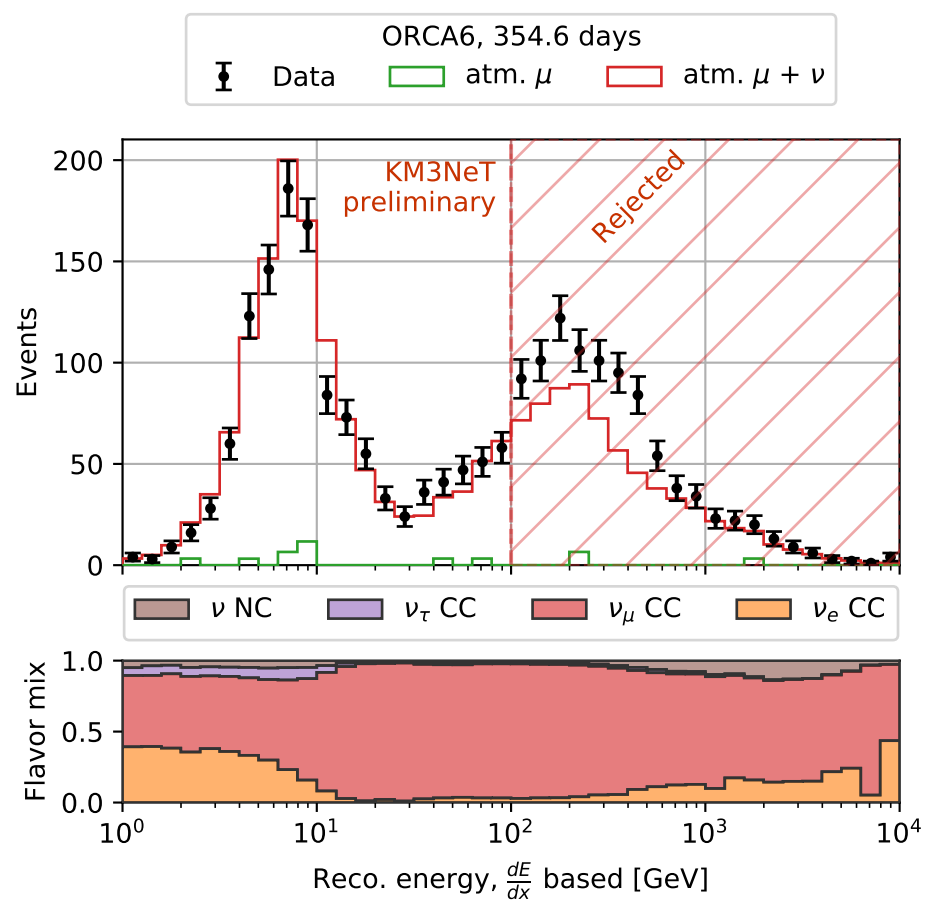

Figure 5: Distribution of the reconstructed energy for data and simulated events after applying all the selection cuts. The flavor mix for the atmospheric neutrino simulated events is also depicted for each energy bin.

The number of data events surviving the selection criteria and the reconstructed enegy cut is 1247, while $1240 \pm 35$ events are expected. This simulated neutrino sample consists of 223 $v_{e}+\overline{v_{e}}$ CC events, $902 v_{\mu}+\overline{v_{\mu}}$ CC events, $70 v_{\tau}+\overline{v_{\tau}}$ CC events as well as $45 \mathrm{NC}$ events. A small contamination of simulated atmospheric muon events is expected, as $31 \pm 10$ events are expected to survive the selection criteria.

The distribution of the cosine of the reconstructed zenith angle is shown in Fig. 6 for the events that survive the pre-selection likelihood cut, as well as for the events that survive all selection criteria and the reconstructed energy cut. In Fig. 7, the detection rate for the selected events is presented as a function of time, accompanied by the respective residuals. 


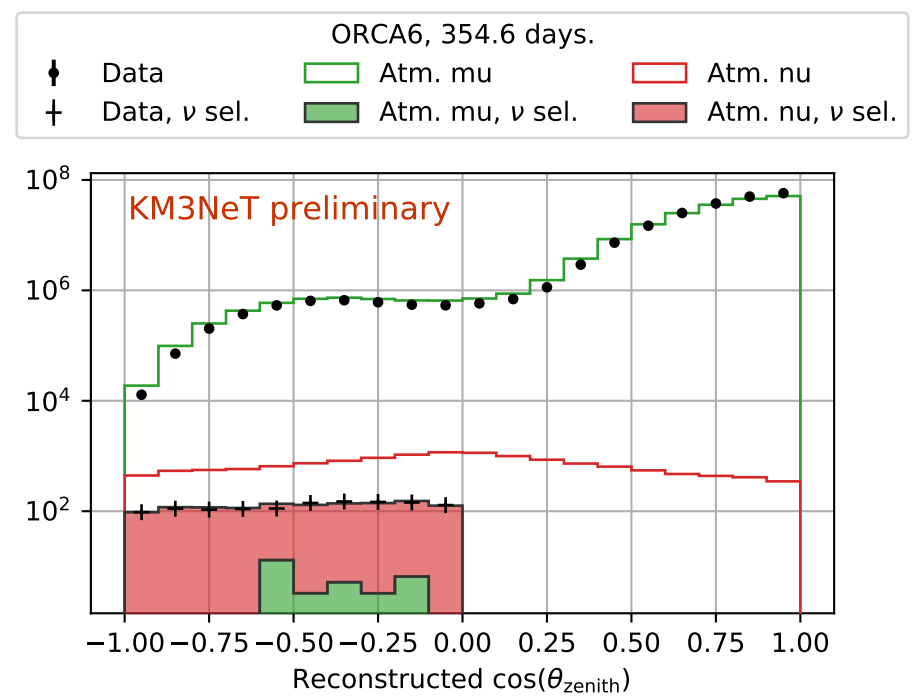

Figure 6: Distribution of the cosine of the reconstructed zenith angle for data and simulated events, after the pre-selection and for the final selection cuts.

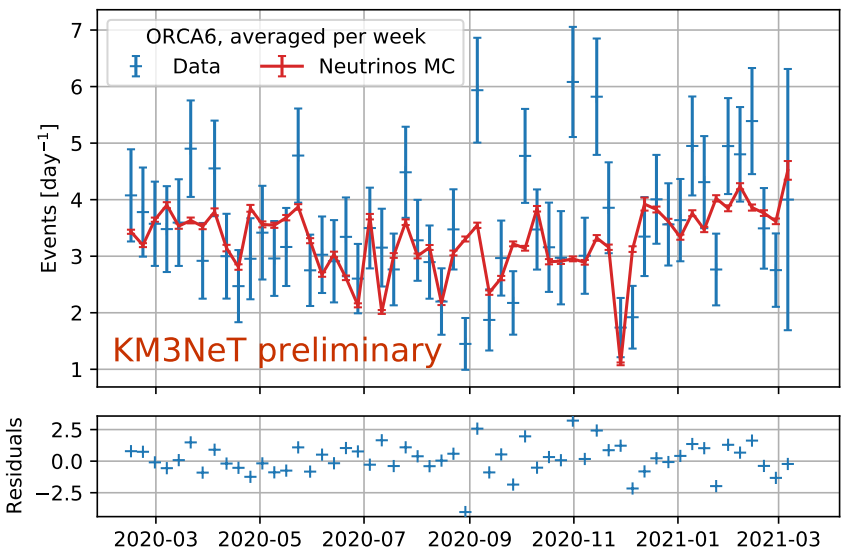

Figure 7: Selected events per day (averaged per week) for data and simulated events along with the respective residuals.

\section{Resolution and prospects}

The selected Monte Carlo (MC) neutrino events illustrate a good reconstruction performance. The angular resolution is presented in Fig. 8, as a function of the simulated neutrino energy. The angular resolution is better than $20^{\circ}$ for energies greater than $4 \mathrm{GeV}$ and better than $10^{\circ}$ for energies greater than $20 \mathrm{GeV}$. 


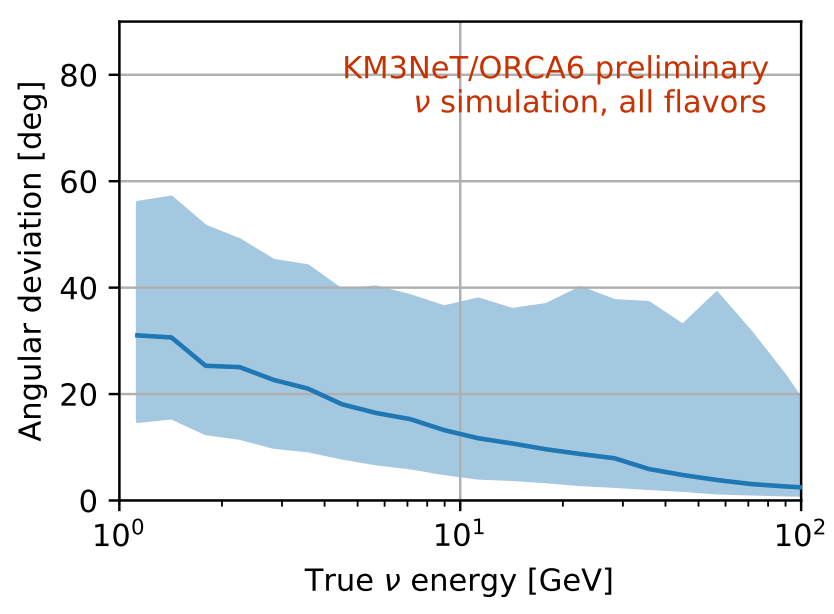

Figure 8: Angular resolution as a function of the neutrino energy for the MC selected neutrino events. The angular resolution is better than $10^{\circ}$ for energies greater than $20 \mathrm{GeV}$. The band represents $\pm 1 \sigma$ uncertainty.

The relationship between the true and the reconstructed energy is described in Fig. 9, where two different curves are shown. The first one represents the neutrino energy estimation using the detected light in a cylinder centred to the reconstructed track (blue). The other (orange) represents the energy estimation using the length of the reconstructed track multiplied by the expected energy loss per meter.

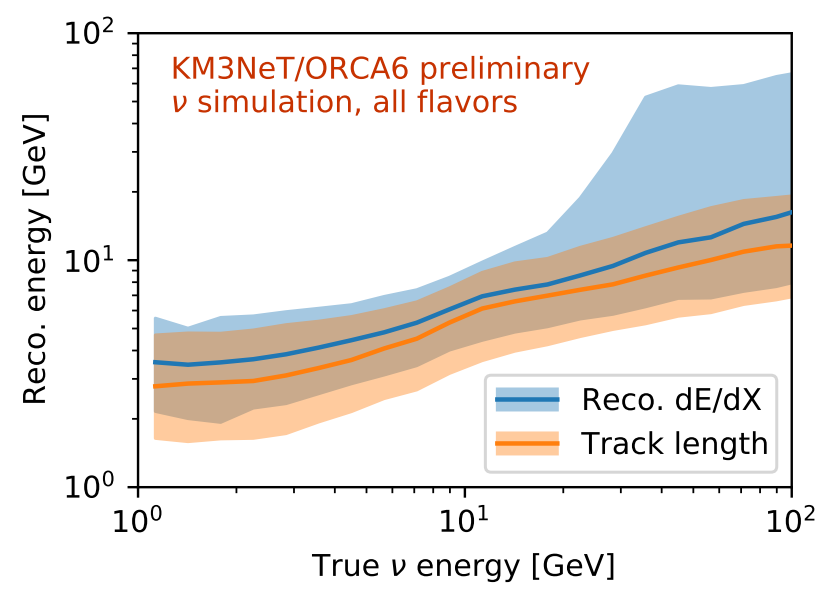

Figure 9: Median reconstructed energy as a function of the neutrino energy for the MC selected events. The bands represent $\pm 1 \sigma$ uncertainty.

The neutrino energy reconstruction performance of the current detector configuration is constrained by its limited size. The upcoming deployments of more detection units will lead to an increase of the energy range and an improvement of the energy reconstruction, as longer tracks will be reconstructed more accurately and a larger fraction of fully contained events will be detected. This improvement will be an important step towards the measurement of the atmospheric neutrino flux. Such a measurement is important in order to test the Cosmic Ray models. The ORCA detector 
is sensitive to an energy range $(1-100 \mathrm{GeV})$ in which there is room for improvement with respect to current experiments. As it is shown in Fig. 10, there exist few measurements especially between 10 and $100 \mathrm{GeV}$.

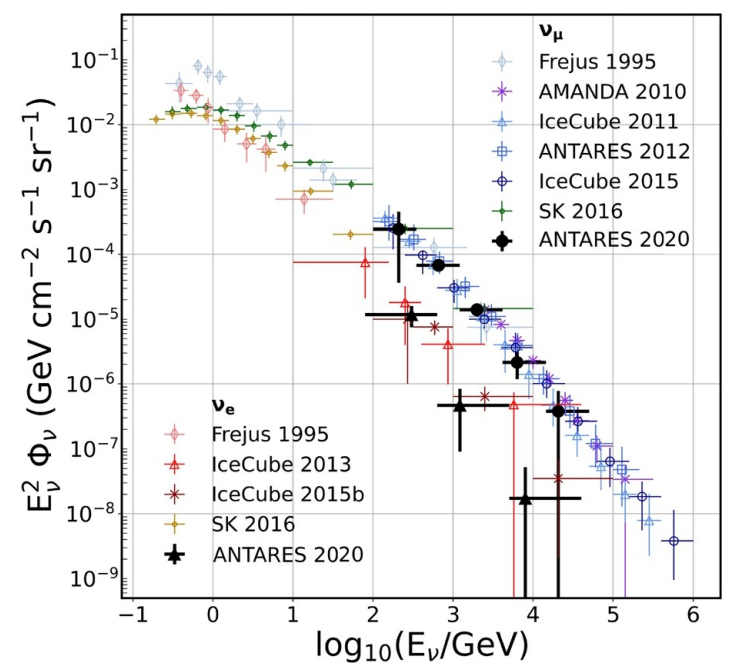

Figure 10: Measurements of the atmospheric neutrino flux by several experiments [8].

\section{Conclusions}

An analysis of ORCA6 data which results in a neutrino sample of high purity has been presented. The data period analysed corresponds to 354.6 days, with a $92 \%$ time efficiency with respect to the full-time period. The application of several selection criteria leads to 1247 events in data, while $1240 \pm 35$ events are expected from the simulation of atmospheric neutrinos and $31 \pm 10$ events from the atmospheric muon simulation. The angular resolution for the expected neutrinos has also been presented, demonstrating a good performance with respect to the early phase of construction. The current performance of the energy reconstruction has also been discussed. This is expected to improve considerably as more detection units are deployed.

\section{References}

[1] S. Adrián-Martínez et al. (KM3NeT Collaboration), 2016 J. Phys. G43084001.

[2] E. Leonora on behalf of the KM3NeT Collaboration 2018 J. Phys.: Conf. Ser.1056 012031.

[3] K. Melis, A. Heijboer and M. de Jong (for the KM3NeT Collaboration), PoS(ICRC2017)950.

[4] S. Aiello et al. (KM3NeT Collaboration), Comput. Phys. Commun. 256, 107477 (2020).

[5] M. Honda et al., Phys. Rev. D 92, 023004 (2015).

[6] JHEP 09 (2020) 178 [arXiv:2007.14792], NuFIT 5.0 (2020), www.nu-fit.org.

[7] G. Carminati, A. Margiotta, M. Spurio, Comput. Phys. Commun.179, 915 (2008).

[8] A. Albert et al. (ANTARES), Phys. Lett. B 816, 136228 (2021). 


\section{Full Author List: KM3NeT Collaboration}

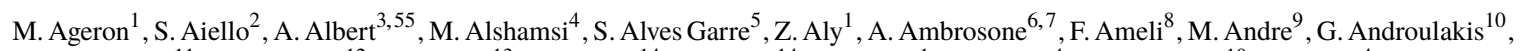
M. Anghinolfi1 ${ }^{11}$, M. Anguita ${ }^{12}$, G. Anton ${ }^{13}$, M. Ardid ${ }^{14}$, S. Ardid $^{14}$, W. Assal ${ }^{1}$, J. Aublin ${ }^{4}$, C. Bagatelas ${ }^{10}$, B. Baret ${ }^{4}$, S. Basegmez du Pree ${ }^{15}$, M. Bendahman ${ }^{4}, 16$, F. Benfenati ${ }^{17,18}$, E. Berbee ${ }^{15}$, A. M. van den Berg ${ }^{19}$, V. Bertin ${ }^{1}$, S. Beurthey ${ }^{1}$, V. van Beveren ${ }^{15}$, S. Biagi ${ }^{20}$, M. Billault ${ }^{1}$, M. Bissinger ${ }^{13}$, M. Boettcher ${ }^{21}$, M. Bou Cabo ${ }^{22}$, J. Boumaaza ${ }^{16}$, M. Bouta ${ }^{23}$, C. Boutonnet ${ }^{4}$, G. Bouvet ${ }^{24}$, M. Bouwhuis ${ }^{15}$, C. Bozza ${ }^{25}$, H.Brânzaş ${ }^{26}$, R. Bruijn ${ }^{15,27}$, J. Brunner ${ }^{1}$, R. Bruno ${ }^{2}$, E. Buis ${ }^{28}$, R. Buompane ${ }^{6,29}$, J. Busto ${ }^{1}$, B. Caiffi ${ }^{11}$, L. Caillat ${ }^{1}$, D. Calvo ${ }^{5}$, S. Campion ${ }^{30,8}$, A. Capone ${ }^{30,8}$, H. Carduner ${ }^{24}$, V. Carretero ${ }^{5}$, P. Castaldi ${ }^{17,31}$, S. Celli ${ }^{30,8}$, R. Cereseto ${ }^{11}$, M. Chabab ${ }^{32}$, C. Champion ${ }^{4}$, N. Chau ${ }^{4}$, A. Chen ${ }^{33}$, S. Cherubini ${ }^{20,34}$, V. Chiarella ${ }^{35}$, T. Chiarusi ${ }^{17}$, M. Circella ${ }^{36}$, R. Cocimano ${ }^{20}$, J. A. B. Coelho ${ }^{4}$, A. Coleiro ${ }^{4}$, M. Colomer Molla ${ }^{4,5}$, S. Colonges ${ }^{4}$, R. Coniglione ${ }^{20}$, A. Cosquer ${ }^{1}$, P. Coyle ${ }^{1}$, M. Cresta ${ }^{11}$, A. Creusot $^{4}$, A. $\mathrm{Cruz}^{37}$, G. Cuttone $^{20}$, A. D'Amico ${ }^{15}$, R. Dallier ${ }^{24}$, B. De Martino ${ }^{1}$, M. De Palma ${ }^{36,38}$, I. Di Palma ${ }^{30,8}$, A.F. Díaz ${ }^{12}$, D. Diego$\operatorname{Tortosa}^{14}$, C. Distefano ${ }^{20}$, A. Domi ${ }^{15,27}$, C. Donzaud ${ }^{4}$, D. Dornic ${ }^{1}$, M. Dörr ${ }^{39}$, D. Drouhin ${ }^{3,55}$, T. Eberl ${ }^{13}$, A. Eddyamoui ${ }^{16}$, T. van Eeden ${ }^{15}$, D. van Eijk ${ }^{15}$, I. El Bojaddaini ${ }^{23}$, H. Eljarrari ${ }^{16}$, D. Elsaesser ${ }^{39}$, A. Enzenhöfer ${ }^{1}$, V. Espinosa ${ }^{14}$, P. Fermani ${ }^{30,8}$, G. Ferrara $^{20,34}$, M. D. Filipovic ${ }^{40}$, F. Filippini ${ }^{17,18}$, J. Fransen ${ }^{15}$, L. A. Fusco ${ }^{1}$, D. Gajanana ${ }^{15}$, T. Gal ${ }^{13}$, J. García Méndez ${ }^{14}$, A. Garcia Soto $^{5}$, E. Garçon ${ }^{1}$, F. Garufi ${ }^{6,7}$, C. Gatius ${ }^{15}$, N. Geißelbrecht ${ }^{13}$, L. Gialanella 6 ,29, E. Giorgio ${ }^{20}$, S. R. Gozzini ${ }^{5}$, R. Gracia ${ }^{15}$, K. Graf ${ }^{13}$, G. Grella ${ }^{41}$, D. Guderian ${ }^{56}$, C. Guidi ${ }^{11,42}$, B. Guillon ${ }^{43}$, M. Gutiérrez ${ }^{44}$, J. Haefner ${ }^{13}$, S. Hallmann ${ }^{13}$, H. Hamdaoui ${ }^{16}$, H. van Haren ${ }^{45}$, A. Heijboer ${ }^{15}$, A. Hekalo ${ }^{39}$, L. Hennig ${ }^{13}$, S. Henry ${ }^{1}$, J. J. Hernández-Rey ${ }^{5}$, J. Hofestädtt ${ }^{13}$, F. Huang ${ }^{1}$, W. Idrissi Ibnsalih ${ }^{6,29}$, A. Ilioni ${ }^{4}$, G. Illuminati ${ }^{17,18,4}$, C. W. James ${ }^{37}$, D. Janezashvili ${ }^{46}$, P. Jansweijer ${ }^{15}$, M. de Jong ${ }^{15,47}$, P. de Jong ${ }^{15,27}$, B. J. Jung ${ }^{15}$, M. Kadler ${ }^{39}$, P. Kalaczyński ${ }^{48}$, O. Kalekin ${ }^{13}$, U. F. Katz ${ }^{13}$, F. Kayzel ${ }^{15}$, P. Keller ${ }^{1}$, N. R. Khan Chowdhury ${ }^{5}$, G. Kistauri ${ }^{46}$, F. van der Knaap ${ }^{28}$, P. Kooijman ${ }^{27,57}$, A. Kouchner ${ }^{4,49}$, M. Kreter ${ }^{21}$, V. Kulikovskiy ${ }^{11}$, M. Labalme ${ }^{43}$, P. Lagier ${ }^{1}$, R. Lahmann ${ }^{13}$, P. Lamare ${ }^{1}$, M. Lamoureux $^{14}$, G. Larosa ${ }^{20}$, C. Lastoria ${ }^{1}$, J. Laurence ${ }^{1}$, A. Lazo ${ }^{5}$, R. Le Breton ${ }^{4}$, E. Le Guirriec ${ }^{1}$, S. Le Stum ${ }^{1}$, G. Lehaut ${ }^{43}$, O. Leonardi ${ }^{20}$, F. Leone ${ }^{20,34}$, E. Leonora ${ }^{2}$, C. Lerouvillois ${ }^{1}$, J. Lesrel ${ }^{4}$, N. Lessing ${ }^{13}$, G. Levi ${ }^{17,18}$, M. Lincetto ${ }^{1}$, M. Lindsey Clark ${ }^{4}$, T. Lipreau ${ }^{24}$, C. LLorens Alvarez ${ }^{14}$, A. Lonardo ${ }^{8}$, F. Longhitano ${ }^{2}$, D. Lopez-Coto ${ }^{44}$, N. Lumb ${ }^{1}$, L. Maderer ${ }^{4}$, J. Majumdar ${ }^{15}$, J. Mańczak ${ }^{5}$, A. Margiotta ${ }^{17,18}$, A. Marinelli ${ }^{6}$, A. Marini ${ }^{1}$, C. Markou ${ }^{10}$, L. Martin ${ }^{24}$, J. A. Martínez-Mora ${ }^{14}$, A. Martini ${ }^{35}$, F. Marzaioli ${ }^{6,29}$,

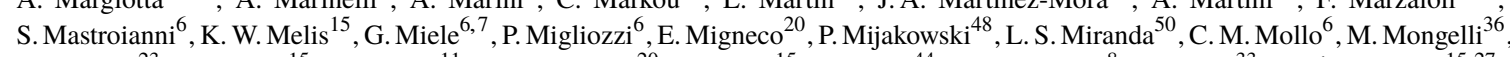
A. Moussa ${ }^{23}$, R. Muller ${ }^{15}$, P. Musico ${ }^{11}$, M. Musumeci ${ }^{20}$, L. Nauta ${ }^{15}$, S. Navas ${ }^{44}$, C. A. Nicolau ${ }^{8}$, B. Nkosi ${ }^{33}$, B. Ó Fearraigh ${ }^{15,27}$, M. O'Sullivan ${ }^{37}$, A. Orlando ${ }^{20}$, G. Ottonello ${ }^{11}$, S. Ottonello ${ }^{11}$, J. Palacios González ${ }^{5}$, G. Papalashvili ${ }^{46}$, R. Papaleo ${ }^{20}$, C. Pastore ${ }^{36}$, A. M. Păun ${ }^{26}$, G.E. Păvălaş ${ }^{26}$, G. Pellegrini ${ }^{17}$, C. Pellegrino ${ }^{18,58}$, M. Perrin-Terrini ${ }^{1}$, V. Pestel ${ }^{15}$, P. Piattelli ${ }^{20}$, C. Pieterse ${ }^{5}$, O. Pisanti ${ }^{6,7}$, C. Poire ${ }^{14}$, V. Popa $^{26}$, T. Pradier ${ }^{3}$, F. Pratolongo ${ }^{11}$, I. Probst ${ }^{13}$, G. Pühlhofer ${ }^{51}$, S. Pulvirenti ${ }^{20}$, G. Quéméner ${ }^{43}$, N. Randazzo ${ }^{2}$, A. Rapicavoli ${ }^{34}$, S. Razzaque ${ }^{50}$, D. Real ${ }^{5}$, S. Reck $^{13}$, G. Riccobene ${ }^{20}$, L. Rigalleau ${ }^{24}$, A. Romanov ${ }^{11,42}$, A. Rovelli ${ }^{20}$, J. Royon $^{1}$, F. Salesa Greus 5 , D. F. E. Samtleben ${ }^{15,47}$, A. Sánchez Losa ${ }^{36,5}$, M. Sanguineti ${ }^{11,42}$, A. Santangelo ${ }^{51}$, D. Santonocito ${ }^{20}$, P. Sapienza $^{20}$, J. Schmelling ${ }^{15}$, J. Schnabel ${ }^{13}$, M. F. Schneider ${ }^{13}$, J. Schumann ${ }^{13}$, H. M. Schutte ${ }^{21}$, J. Seneca ${ }^{15}$, I. Sgura ${ }^{36}$, R. Shanidze ${ }^{46}$, A. Sharma ${ }^{52}$, A. Sinopoulou ${ }^{10}$, B. Spisso ${ }^{41,6}$, M. Spurio ${ }^{17,18}$, D. Stavropoulos ${ }^{10}$, J. Steijger ${ }^{15}$, S. M. Stellacci ${ }^{41,6}$, M. Taiuti ${ }^{11,42}$, F. Tatone ${ }^{36}$, Y. Tayalati ${ }^{16}$, E. Tenllado ${ }^{44}$, D. Tézier ${ }^{1}$, T. Thakore ${ }^{5}$, S. Theraube ${ }^{1}$, H. Thiersen $^{21}$, P. Timmer $^{15}$, S. Tingay ${ }^{37}$, S. Tsagkli $^{10}$, V. Tsourapis ${ }^{10}$, E. Tzamariudaki ${ }^{10}$, D. Tzanetatos ${ }^{10}$, C. Valieri ${ }^{17}$, V. Van Elewyck ${ }^{4,49}$, G. Vasileiadis ${ }^{53}$, F. Versari ${ }^{17,18}$, S. Viola ${ }^{20}$, D. Vivolo ${ }^{6,29}$, G. de Wasseige $^{4}$, J. Wilms ${ }^{54}$, R. Wojaczyński ${ }^{48}$, E. de Wolf ${ }^{15,27}$, T. Yousfi ${ }^{23}$, S. Zavatarelli ${ }^{11}$, A. Zegarelli ${ }^{30,8}$, D. Zito ${ }^{20}$, J. D. Zornoza ${ }^{5}$, J. Zúñiga ${ }^{5}$, N. Zywucka ${ }^{21}$.

${ }^{1}$ Aix Marseille Univ, CNRS/IN2P3, CPPM, Marseille, France.

${ }^{2}$ INFN, Sezione di Catania, Via Santa Sofia 64, Catania, 95123 Italy.

${ }^{3}$ Université de Strasbourg, CNRS, IPHC UMR 7178, F-67000 Strasbourg, France.

${ }^{4}$ Université de Paris, CNRS, Astroparticule et Cosmologie, F-75013 Paris, France.

${ }^{5}$ IFIC - Instituto de Física Corpuscular (CSIC - Universitat de València), c/Catedrático José Beltrán, 2, 46980 Paterna, Valencia, Spain. ${ }^{6}$ INFN, Sezione di Napoli, Complesso Universitario di Monte S. Angelo, Via Cintia ed. G, Napoli, 80126 Italy.

${ }^{7}$ Università di Napoli “Federico II”, Dip. Scienze Fisiche "E. Pancini”, Complesso Universitario di Monte S. Angelo, Via Cintia ed. G, Napoli, 80126 Italy.

${ }^{8}$ INFN, Sezione di Roma, Piazzale Aldo Moro 2, Roma, 00185 Italy.

${ }^{9}$ Universitat Politècnica de Catalunya, Laboratori d'Aplicacions Bioacústiques, Centre Tecnològic de Vilanova i la Geltrú, Avda. Rambla Exposició, s/n, Vilanova i la Geltrú, 08800 Spain.

${ }^{10}$ NCSR Demokritos, Institute of Nuclear and Particle Physics, Ag. Paraskevi Attikis, Athens, 15310 Greece.

${ }^{11}$ INFN, Sezione di Genova, Via Dodecaneso 33, Genova, 16146 Italy.

${ }^{12}$ University of Granada, Dept. of Computer Architecture and Technology/CITIC, 18071 Granada, Spain.

${ }^{13}$ Friedrich-Alexander-Universität Erlangen-Nürnberg, Erlangen Centre for Astroparticle Physics, Erwin-Rommel-Straße 1, 91058 Erlangen, Germany.

${ }^{14}$ Universitat Politècnica de València, Instituto de Investigación para la Gestión Integrada de las Zonas Costeras, C/ Paranimf, 1, Gandia, 46730 Spain.

${ }^{15}$ Nikhef, National Institute for Subatomic Physics, PO Box 41882, Amsterdam, 1009 DB Netherlands.

${ }^{16}$ University Mohammed V in Rabat, Faculty of Sciences, 4 av. Ibn Battouta, B.P. 1014, R.P. 10000 Rabat, Morocco.

${ }^{17}$ INFN, Sezione di Bologna, v.le C. Berti-Pichat, 6/2, Bologna, 40127 Italy.

${ }^{1}$ also at Dipartimento di Fisica, INFN Sezione di Padova and Università di Padova, I-35131, Padova, Italy 
${ }^{18}$ Università di Bologna, Dipartimento di Fisica e Astronomia, v.le C. Berti-Pichat, 6/2, Bologna, 40127 Italy.

${ }^{19}$ KVI-CART University of Groningen, Groningen, the Netherlands.

${ }^{20}$ INFN, Laboratori Nazionali del Sud, Via S. Sofia 62, Catania, 95123 Italy.

${ }^{21}$ North-West University, Centre for Space Research, Private Bag X6001, Potchefstroom, 2520 South Africa.

${ }^{22}$ Instituto Español de Oceanografía, Unidad Mixta IEO-UPV, C/ Paranimf, 1, Gandia, 46730 Spain.

${ }^{23}$ University Mohammed I, Faculty of Sciences, BV Mohammed VI, B.P. 717, R.P. 60000 Oujda, Morocco.

${ }^{24}$ Subatech, IMT Atlantique, IN2P3-CNRS, Université de Nantes, 4 rue Alfred Kastler - La Chantrerie, Nantes, BP 2072244307 France.

${ }^{25}$ Università di Salerno e INFN Gruppo Collegato di Salerno, Dipartimento di Matematica, Via Giovanni Paolo II 132, Fisciano, 84084 Italy.

${ }^{26}$ ISS, Atomistilor 409, Măgurele, RO-077125 Romania.

${ }^{27}$ University of Amsterdam, Institute of Physics/IHEF, PO Box 94216, Amsterdam, 1090 GE Netherlands.

${ }^{28}$ TNO, Technical Sciences, PO Box 155, Delft, 2600 AD Netherlands.

${ }^{29}$ Università degli Studi della Campania "Luigi Vanvitelli", Dipartimento di Matematica e Fisica, viale Lincoln 5, Caserta, 81100 Italy.

${ }^{30}$ Università La Sapienza, Dipartimento di Fisica, Piazzale Aldo Moro 2, Roma, 00185 Italy.

${ }^{31}$ Università di Bologna, Dipartimento di Ingegneria dell'Energia Elettrica e dell'Informazione "Guglielmo Marconi", Via dell'Università 50, Cesena, 47521 Italia.

${ }^{32}$ Cadi Ayyad University, Physics Department, Faculty of Science Semlalia, Av. My Abdellah, P.O.B. 2390, Marrakech, 40000 Morocco.

${ }^{33}$ University of the Witwatersrand, School of Physics, Private Bag 3, Johannesburg, Wits 2050 South Africa.

${ }^{34}$ Università di Catania, Dipartimento di Fisica e Astronomia "Ettore Majorana", Via Santa Sofia 64, Catania, 95123 Italy.

${ }^{35}$ INFN, LNF, Via Enrico Fermi, 40, Frascati, 00044 Italy.

${ }^{36}$ INFN, Sezione di Bari, via Orabona, 4, Bari, 70125 Italy.

${ }^{37}$ International Centre for Radio Astronomy Research, Curtin University, Bentley, WA 6102, Australia.

${ }^{38}$ University of Bari, Via Amendola 173, Bari, 70126 Italy.

${ }^{39}$ University Würzburg, Emil-Fischer-Straße 31, Würzburg, 97074 Germany.

${ }^{40}$ Western Sydney University, School of Computing, Engineering and Mathematics, Locked Bag 1797, Penrith, NSW 2751 Australia.

${ }^{41}$ Università di Salerno e INFN Gruppo Collegato di Salerno, Dipartimento di Fisica, Via Giovanni Paolo II 132, Fisciano, 84084 Italy.

${ }^{42}$ Università di Genova, Via Dodecaneso 33, Genova, 16146 Italy.

${ }^{43}$ Normandie Univ, ENSICAEN, UNICAEN, CNRS/IN2P3, LPC Caen, LPCCAEN, 6 boulevard Maréchal Juin, Caen, 14050 France.

${ }^{44}$ University of Granada, Dpto. de Física Teórica y del Cosmos \& C.A.F.P.E., 18071 Granada, Spain.

${ }^{45}$ NIOZ (Royal Netherlands Institute for Sea Research), PO Box 59, Den Burg, Texel, 1790 AB, the Netherlands.

${ }^{46}$ Tbilisi State University, Department of Physics, 3, Chavchavadze Ave., Tbilisi, 0179 Georgia.

${ }^{47}$ Leiden University, Leiden Institute of Physics, PO Box 9504, Leiden, 2300 RA Netherlands.

${ }^{48}$ National Centre for Nuclear Research, 02-093 Warsaw, Poland.

${ }^{49}$ Institut Universitaire de France, 1 rue Descartes, Paris, 75005 France.

${ }^{50}$ University of Johannesburg, Department Physics, PO Box 524, Auckland Park, 2006 South Africa.

${ }^{51}$ Eberhard Karls Universität Tübingen, Institut für Astronomie und Astrophysik, Sand 1, Tübingen, 72076 Germany.

${ }^{52}$ Università di Pisa, Dipartimento di Fisica, Largo Bruno Pontecorvo 3, Pisa, 56127 Italy.

${ }^{53}$ Laboratoire Univers et Particules de Montpellier, Place Eugène Bataillon - CC 72, Montpellier Cédex 05, 34095 France.

${ }^{54}$ Friedrich-Alexander-Universität Erlangen-Nürnberg, Remeis Sternwarte, Sternwartstraße 7, 96049 Bamberg, Germany.

${ }^{55}$ Université de Haute Alsace, 68100 Mulhouse Cedex, France.

${ }^{56}$ University of Münster, Institut für Kernphysik, Wilhelm-Klemm-Str. 9, Münster, 48149 Germany.

${ }^{57}$ Utrecht University, Department of Physics and Astronomy, PO Box 80000, Utrecht, 3508 TA Netherlands.

${ }^{58}$ INFN, CNAF, v.le C. Berti-Pichat, 6/2, Bologna, 40127 Italy. 